\title{
Dentatorubropallidoluysian atrophy in a Spanish family: a clinical, radiological, pathological, and genetic study
}

\author{
E Muñoz, M Milà, A Sánchez, P Latorre, A Ariza, M Codina, F Ballesta, E Tolosa
}

Department of

Neurology

E Muñoz

E Tolosa

Department of Genetics, Hospital

Clínic i Universitari, Barcelona, Spain

$M$ Milà

A Sánchez

F Ballesta

\section{Department of \\ Neurology \\ P Latorre \\ M Codina}

Department of Pathology, Hospital Universitari Germans

Trias i Pujol, Badalona, Spain A Ariza

Correspondence to: Dr Esteban Muñoz, Department of Neurology, Hospital Clínic i Universitari, Villarroel 170, 08036 Barcelona, Spain. Telephone 00349322754 14; fax 0034

9322757 83; email: jmunozg@meditex.es

Received 15 January 1999 and in revised form 12 April 1999

Accepted 9 June 1999

\section{Abstract}

The object was to describe the clinical, radiological, pathological, and genetic findings in a Spanish family with dentatorubropallidoluysian atrophy (DRPLA). This is an inherited neurodegenerative disease, well recognised in Japan, but with few cases reported from Europe and America and no cases published from Spain. The clinical misdiagnosis of Huntington's disease is not infrequent.

Pedigree analysis and clinical data of a family were collected. A genetic study was performed in two patients. Pathological information was obtained from the necropsy of one patient.

Results-Pedigree analysis showed an autosomal dominant pattern of inheritance. Age at onset varied from 5 to 55 years. Ataxia and chorea were present in most of the members. Some of these had a long course disease with late dementia. Four patients had seizures and early mental impairment. In one patient, cranial MRI showed cortical, brain stem and cerebellar atrophy, and white matter changes. In another patient, necropsy showed atrophy of the globus pallidus and lipofuscin deposits in dentate and pallidal neuronal cells. Genetic study showed an abnormal CAG triplet expansion in the $B 37$ gene on chromosome 12 .

As in other cases previously reported, Spanish cases of DRPLA show intrafamilial phenotypic heterogeneity. Clinical and MRI data could differentiate DRPLA from Huntington's disease but definitive diagnosis requires molecular studies. Pathological studies are still necessary to correlate DRPLA brain involvement with the clinical and molecular findings. (F Neurol Neurosurg Psychiatry 1999;67:811-814)

Keywords: dentatorubropallidoluysian atrophy; genetics; Huntington's disease; spinocerebellar ataxia

Dentatorubropallidoluysian atrophy (DRPLA) is a neurodegenerative disease characterised mainly by cerebellar ataxia, choreoathetosis, myoclonus, dementia, and behavioural disturbances. ${ }^{1-8}$ Its estimated prevalence in the
Japanese population is 0.2 to 0.7 per $100000,^{3}$ but only few families have been reported outside Japan. ${ }^{4-8}$ The pattern of inheritance is autosomal dominant and the genetic defect consists of an expansion of unstable trinucleotide (CAG) repeats in the $B 37$ gene on the short arm of chromosome $12 .^{9}{ }^{10}$ We report the clinical, radiological, pathological, and genetic findings of a Spanish family with the diagnosis of DRPLA.

\section{Materials and methods}

Clinical information was collected from a family whose pedigree suggested an autosomal dominant inherited disease.

Genomic DNA was obtained from peripheral blood leucocytes in two patients (III-7 and IV-4) after standard procedures. A polymerase chain reaction (PCR) was performed using the primer set described by Koide et al for the identification of DRPLA alleles.

Macroscopic and microscopic pathological information was obtained from the revised necropsy of one patient (III-5), who died in 1988.

\section{Results}

Twelve members from the family were affected by the disease. Age at onset varied from 5 to 55 years. The main clinical picture in this family was dominated by ataxia, choreoathetosis, behavioural disturbances, and mental impairment (table). Moreover, three members, with childhood onset, showed mental deterioration and epilepsy. Genetic study on patients III-7 and IV-4 confirmed the diagnosis of DRPLA.

\section{CASE REPORTS}

Patient III-7

This 63 year old woman began her illness at the age of 27 when she became irritable and depressed. Six years later she developed gait instability that worsened slowly through the years. At the age of 50 she needed assistance when walking in the street and presented a slurred speech. Two years later the patient's memory began to decline and her personal appearance became negligent and untidy. Cognitive and behavioural changes worsened and at the age of 60 she was unable to dress or feed herself. At this time, she presented a marked gait 
Clinical features of the members affected with presumed DRPLA

\begin{tabular}{|c|c|c|c|c|c|c|c|c|c|c|}
\hline Patient & $\begin{array}{l}\text { Age at } \\
\text { onset }\end{array}$ & $\begin{array}{l}\text { Current } \\
\text { age }\end{array}$ & $\begin{array}{l}\text { Age of } \\
\text { death }\end{array}$ & Cause of death & Ataxia & Chorea & Behavioural disturbances & Dementia & Seizures & Myoclonus \\
\hline $\mathrm{II}-2$ & UK & & 63 & Lung cancer & + & - & Irritability, dysphoria & - & - & - \\
\hline II-4 & 39 & & 65 & Prostate cancer & + & + & Irritability, bad temper & - & - & UK \\
\hline II-5 & 25 & & 50 & Disease progression & + & + & Apathy & + & - & UK \\
\hline III-2 & 50 & 65 & & & + & + & - & - & - & - \\
\hline III-5 & 31 & & 44 & Pneumonia & + & + & Dysphoria & + & + & + \\
\hline III-7 & 27 & 63 & & & + & + & Irritability, depression & + & - & - \\
\hline III-8 & 55 & 60 & & & + & - & Irritability, anxiety & - & - & - \\
\hline III-9 & UK & & 45 & Disease progression & + & + & Psychosis & + & - & UK \\
\hline III-11 & 5 & & 11 & Disease progression & + & - & UK & + & + & + \\
\hline IV-2 & 10 & 20 & & & + & + & & + & + & UK \\
\hline IV-4 & 30 & 32 & & & + & - & Depression, anxiety & - & - & - \\
\hline IV-7 & 5 & 16 & & & - & + & & + & + & - \\
\hline
\end{tabular}

+=Presence; $-=$ absence; $\mathrm{UK}=$ unknown or contradictory information.

instability with clumsiness of her limb movements and dysarthria. One month before admission to our hospital the patient experienced a fall with cranial contusion. Subsequently, she presented incontinence of sphincters, was unable to walk even with assistance, and developed persistent insomnia and nocturnal psychomotor agitation.

On admission she was disoriented, with decreased attention. It was impossible to carry out a mini mental state examination. She sometimes did not recognise some family members. Her speech was moderately dysarthric but no important language defects were detected. Ocular motility was without paresis or nystagmus but she had slow saccades and was unable to look in the direction requested without moving her head to the same side. No limb paresis were seen. Deep tendon reflexes were present but symmetrically decreased and plantar responses were in flexion. Symmetric bilateral dysdiadochokinesia and ankle-kneeshin and nose to finger dysmetria was found. Standing up or walking was impossible because of marked instability. She was unable to take a single step, even with assistance. She presented slight choreic head and tongue movements and choreoatethosic movements were sometimes seen when she tried to move her arms but no involuntary limb movements were seen at rest.

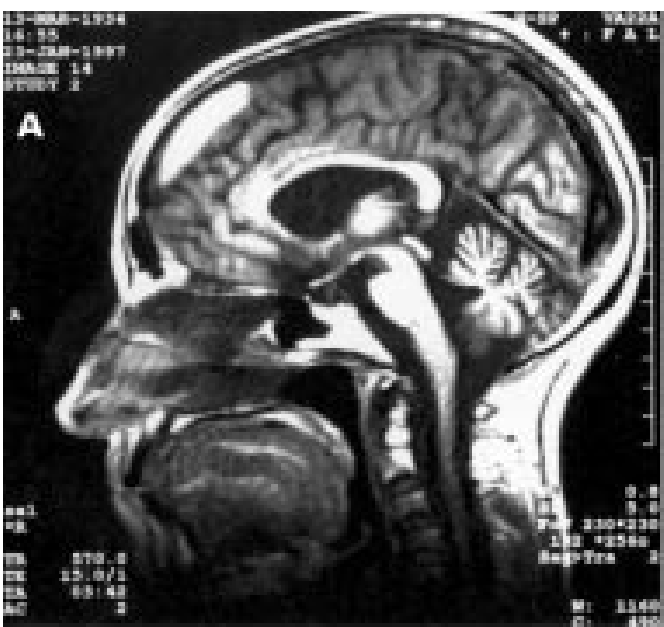

Cranial MRI of patient III-7. (A) T1 weighted image in the sagittal plane showing atrophy of cerebellar vermis and base of the pons. (B) $T 2$ weighted image in the axial plane showing diffuse high intensity signals in the white matter and the presence of a subdural haematoma.
Cranial MRI showed atrophy of the cerebellum, brainstem, and cerebral cortex and many subcortical high intensity lesions in T2 weighted images (figure). In addition, a subdural haematoma on the left cerebral hemisphere was detected.

Molecular study of the B37 gene showed two alleles of 15/60 CAG repeats (normal range: 7-25)

\section{Patient III-5}

This woman was a cousin of patient III-7. Her first neurological symptoms began at the age of 31 with grand mal seizures. At the age of 34 , she began to show behavioural disturbances and 1 year later abnormal involuntary movements of the head and arms were noted. In subsequent years, she showed cognitive deterioration which evolved rapidly. At the age of 37, she began to have trouble walking, with unsteadiness and falls. In 1987, at the age of 41, she was admitted to the hospital. She presented severe cognitive deterioration and depended on others to care for her. She had spontaneous and isolated myoclonus in all limbs and choreic movements of her arms and face. She could not stand or walk without assistance. There was finger-nose and heel-knee ataxia. Deep tendon reflexes were brisk but Babinski's sign was not present. Ocular movements were without ophthalmoparesis

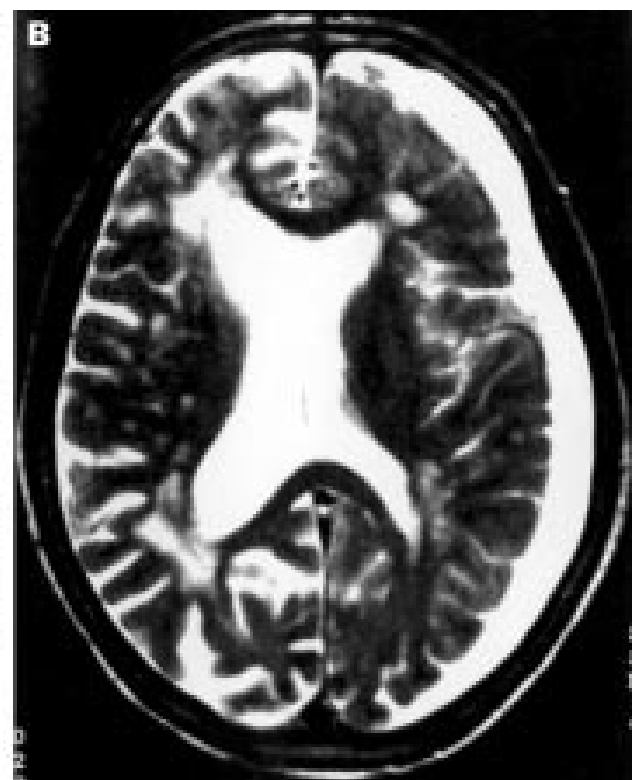


but there was nystagmus to both lateral gazes. Cranial CT showed cortical atrophy, ventricular enlargement, and atrophy of the cerebellar vermis.

The patient died in 1988 because of aspiration pneumonia and necropsy was carried out. The brain were devoid of gross signs of atrophy, except for the globus pallidus, which demonstrated a bilateral atrophic appearance, with its maximum thickness in a coronal plane being $3 \mathrm{~mm}$. On microscopic examination there was a moderate to severe bilateral loss of neurons and reactive gliosis in the external segment of the globus pallidus, where lipofucsin deposition was appreciated within the cells and scattered through the neuropil. The dentate, red, and subthalamic nuclei showed only focal mild neuronal loss. Perikaryal lipofucsin was seen in surviving neurons in the dentate and axonal spheroids were present in the pallidus and dentate but no significant positivity was obtained with immunohistochemistry for ubiquitin.

\section{Discussion}

We describe here the first family with genetically established DRPLA reported in Spain. Most members presented with ataxia and chorea and some of these developed dementia late in the course of the disease. Other members had seizures and early mental impairment, and in three of them the disease began in childhood. Most patients had behavioural disturbances. This familial phenotypic variability has been previously described in other families with DRPLA. ${ }^{25} 6$ Patients with onset in childhood tend to have a myoclonus epilepsy syndrome with progressive mental deterioration, whereas in adults two forms have been described: a pseudoHuntington type characterised by chorea, cognitive dysfunction, and psychiatric symptoms and an ataxochoreoathetoid type characterised by progressive ataxia and choreoathetosis with some dementia. ${ }^{11}$ However, many cases of DRPLA described in the literature present with a mix of symptoms and signs of these three forms. As in other neurodegenerative disorders with expanded trinucleotide repeats, clinical heterogeneity could be related to the number of CAG repeats. The length of CAG repeats in the $B-37$ gene of normal people is generally under 25 , whereas patients with DRPLA have a length greater than 49 expansions. ${ }^{9}{ }^{10} 12$ A correlation between the size of the expanded alleles and the age at onset and the phenotype has been found. Thus patients with progressive myoclonus epilepsy phenotype generally have the largest expansions and the earliest ages of onset. ${ }^{13}{ }^{14}$ However, the relation between the absolute number of repeats and the age of onset and the phenotype in individual cases could be variable. ${ }^{6}$

Before the genetic test, patients III- 5 and III-7 had been diagnosed as having Huntington's disease and familial olivopontocerebellar atrophy (OPCA). But, atypical clinical characteristics for these two different diseases may be considered in these two cases. Huntington's disease begins with behavioural disorders in many cases, but when the disease clinically appears the course is generally more rapidly progressive than in patient III-7 and dementia and/or chorea are the symptoms that usually dominate the clinical picture. ${ }^{15}$ However, in patient III-7, chorea was a minor symptom and dementia appeared late in the course of the disease. Patient III-5 presented with seizures and myoclonus. Although both symptoms have been described in some cases of juvenile Huntington's disease, they are very uncommon in adulthood forms of the disease. ${ }^{15}$ As in other DRPLA cases, MRI findings in our patient III-7 include cerebellar and brain stem atrophy and diffuse high intensity signals in T2 weighted images of cerebral white matter and brain stem (figure). ${ }^{316}$ However, significant cerebellar and brain stem atrophy or white matter changes are very unusual findings in Huntington's disease. ${ }^{16-19}$ In pathologically established familial OPCA ${ }^{20}$ or, more recently, in genetically proved spinocerebellar ataxias (SCA), ${ }^{21}$ the cerebellar dysfunction is the most outstanding clinical feature of the disease but contrary to that reported in DRPLA, mental impairment and abnormal movements such as choreoathetosis or myoclonus are generally not very obvious manifestations in the early stages. By contrast, other manifestations often described in patients with OPCA ${ }^{20}$ or $\mathrm{SCA}^{21}$ such as ophthalmoparesis, and pyramidal or parkinsonian signs are unusual in patients with DRPLA $^{5}$ and were absent in our patients.

We propose that in adult patients with Huntington's disease-like clinical features such as chorea and dementia, the additional presence of a personal or familial cerebellar dysfunction or seizures or a cranial MRI showing pronounced cerebellar or brain stem atrophy or white matter changes might be warning markers for the diagnosis of Huntington's disease. In those patients with autosomal dominant cerebellar ataxia, but with a personal or familial history of seizures, early abnormal movements, or early dementia, the clinical diagnosis of DRPLA might emerge. In all these cases, the genetic test for DRPLA must be considered.

The main pathological changes in DRPLA occur in the dentate nucleus, brainstem, and globus pallidus with neuronal loss, gliosis, and degeneration of the cerebral and cerebellar white matter. The degree of involvement of such structures is variable. ${ }^{19}$ The neuropathological findings in our patient III-5 consisted of degeneration with predominant involvement of the globus pallidus with only mild neuronal loss in the dentate and red nuclei, and lipofuscin deposits. Similar deposits have been also described in other patients with DRPLA ${ }^{511}$ and in other non-lysosomal neurodegenerative diseases. ${ }^{22}{ }^{23}$ It has been suggested that lipofuscin formation in neurons could be due to the alteration of lysosomal function by oxygen derived free radical production. ${ }^{22}$ This mechanism may be integrated in a common pathway for various neurodegenerative disorders, including DRPLA, leading to neuronal death. 
1 Naito H, Oyanagi S. Familial myoclonus epilepsy and choreoathetosis: hereditary dentatorubral-pallidoluysian choreoathetosis: hereditary dentator

2 Takahashi H, Ohama E, Naito $\mathrm{H}$, et al. Hereditary dentotorubral-pallidoluysian atrophy: clinical and pathologic variants in a family. Neurology 1988;38:1065-70.

3 Koide R, Onodera O, Ikeuchi T, et al. Atrophy of the cerebellum and brainstem in dentatorubral pallidoluysian atrophy. Influence of CAG repeat size on MRI findings Neurology 1997;49:1605-12.

4 Warner TT, Williams L, Harding AE. DRPLA in Europe. Nat Genet 1994;6:225.

5 Warner TT, Lennox GG, Janota I, et al. Autosomal-dominat dentatorubropallidoluysian atrophy in the United Kingdom. Mov Disord 1994;9:289-96.

6 Potter NT, Meyer MA, Zimmerman AW, et al. Molecular and clinical findings in a family with dentatorubralpallidoluysian atrophy. Ann Neurol 1995;37:273-7.

7 Burke JR, Wingfield MS, Lewis KE, et al. The Haw River syndrome: Dentatorubropallidoluysian atrophy (DRPLA) in a African-American family. Nat Genet 1994;7:521-4.

8 Nielsen JE, Sorensen K, Hasholt L, et al. Dentatorubralpallidoluysian atrophy. Clinical features of a five-generation pallidoluysian atrophy. Clinical features of a

9 Koide R, Ikeuchi T, Onodera O, et al. Unstable expansion onf CAG repeat in hereditary dentatorubral-pallidoluysian atrophy (DRPLA). Nat Genet 1994;6:9-13.

10 Nagafuchi S, Yanagisawa H, Sato K, et al. Dentatorubral and pallidoluysian atrophy expansion of a unstable CAG trinucleotide on chromosome 12p. Nat Genet 1994;6:14-8.

11 Iizuka R, Hirayama K, Maehara K. Dentato-rubropallidoluysian atrophy: a clinico-pathological study. $\mathcal{f} \mathrm{Neu}-$ rol Neurosurg Psychiatry 1984;47:1288-98.

12 Uyama E, Kondo I, Uchino $\mathrm{M}$, et al. Dentatorubralpallidoluysian atrophy (DRPLA): clinical, genetic, and neuroradiologic studies in a family. $F$ Neurol $S c i 1995 ; 130$ : $146-53$.
13 Ikeuchi $\mathrm{T}$, Koide $\mathrm{R}$, Tanaka $\mathrm{H}$, et al. Dentatorubralpallidoluysian atrophy: clinical features are closely related to unstable expansions of

14 Komure O, Sano A, Nishino N, et al. DNA analysis in hereditary dentatorubral-pallidoluysian atrophy: correlation between CAG repeat length and phenotypic variation and the molecular basis of anticipation. Neurology 1995;45:143-9.

15 Weiner WJ, Lang AE. Huntington's disease. In: Movement disorders. A comprehensive survey. Mount Kisco, New York: Futura, 1989:293-346.

16 Yoshii F, Tomiyasu H, Shinohara Y. Fluid attenuation inversion recovery (FLAIR) images of dentatorubropallidoluysian atrophy: case report. $\mathcal{F}$ Neurol Neurosurg Psychiatry 1998;65:396-9.

17 Castaigne P, Escourolle R, Gray MF. Chorée de Huntington et atrophie cérébelleuse (A propos d'une observation anatomo-clinique). Rev Neurol (Paris) 1976;132:233-40

18 Rodda RA. Cerebellar atrophy in Huntington's disease. 7 Neurol Sci 1981;50:147-57.

19 Becher MW, Rubinsztein DC, Lego J, et al. Dentatorubral and pallidoluysian atrophy (DRPLA). Clinical and neuropathological findings in genetically confirmed North American and European pedigrees. Mov Disord 1997;12: $519-30$

20 Berciano J. Olivopontocerebellar atrophy. A review of 117 cases. F Neurol Sci 1982;53:253-72.

1 Schöls L, Amoiridis G, Büttner T, et al. Autosomal dominant cerebellar ataxia: phenotypic differences in genetically defined subtypes?. Ann Neurol 1997;42:924-32.

22 Nixon RA, Cataldo AM, Paskevich PA, et al. The lysosomal system in neurons. Involvement at multiple stages of Alzheimer's disease pathogenesis. Ann N Y Acad Sci 1992; 674:65-88.

23 Goebel HH, Heipertz R, Scholz W, et al. Juvenile Huntington chorea: clinical, ultrastructural, and biochemical studies. Neurology 1978;28:23-31.

\section{NEUROLOGICAL STAMP}

\section{Neurological Advertisements on Stamps}

In 1893 the New Zealand Government increased its revenue by utilising the backs of stamps for advertising purposes. All denominations from $1 \mathrm{~d}$ to 1 /- were made use of. The non-gummed side of the stamp showed the side face of Queen Victoria. Amongst the advertisements were Beechams Pills for sick headaches, for invigorating the nerves and for nervous ills. Bonnington's Irish Moss was advertised to sustain the use and flexibility of the voice. The advertising was not confined to neurological or medical disorders. A registered medical practitioner, Dr McBean Stewart, advertised his name and his cure for croup, diphtheria and asthma. (Scott 61-67, 68-69).

L F HAAS

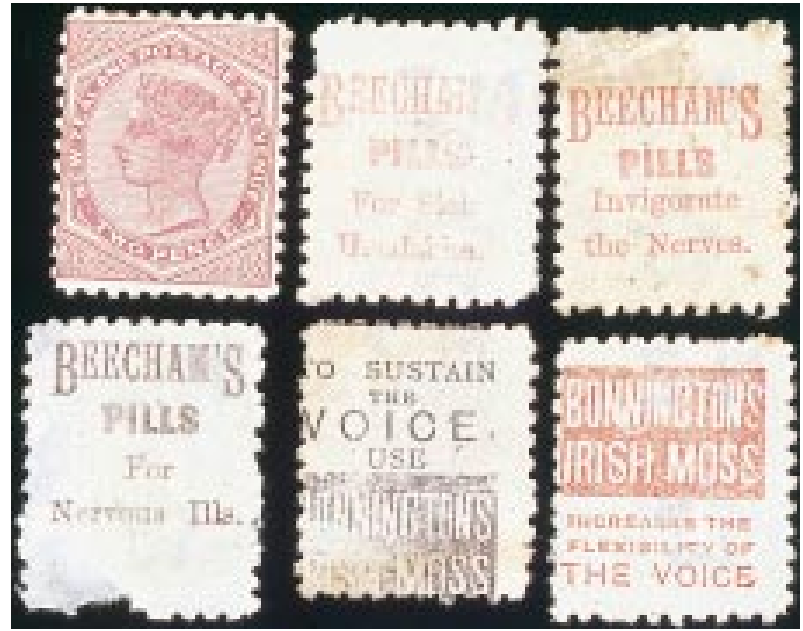

